\title{
How to Prevent COVID-19 in Nurses and Physicians in the Hospital Setting: Universal Test-and-Isolate for All Patients Admitted to Hospitals
}

\author{
Julio A. Ramirez ${ }^{1}{ }^{2 *}, M D ;$ Ruth Carrico ${ }^{1},{ }^{2}$, PhD; Forest W. Arnold ${ }^{1},{ }^{2}, D O ;$ Paul Schulz ${ }^{3}, M D$ \\ ${ }^{1}$ Division of Infectious Diseases, Department of Medicine, School of Medicine, University of Louisville, Louisville, KY, USA; ${ }^{2}$ Center of Excellence for Research in \\ Infectious Diseases, University of Louisville, Louisville, KY, USA; ${ }^{3}$ Infectious Diseases, Norton Healthcare, Louisville, KY, USA \\ *j.ramirez@louisville.edu
}

Recommended Citation: Ramirez JA, Carrico R, Arnold FW, Schulz P. How to prevent COVID-19 in nurses and physicians in the hospital setting: Universal test-and-isolate for all patients admitted to hospitals. Univ Louisville J Respir Infect 2020; 4(1):Article 6. doi: 10.18297/jri/vol4/iss1/6.

The traditional approach to protect healthcare workers (HCWs) from acquiring infections in the hospital setting has been based on isolating patients suspected of having an infection, until the infection is ruled out (e.g. tuberculosis). This approach has been used as a way to protect HCWs from COVID-19. Patients suspected of having COVID-19 are placed in contact and airborne isolation until the infection is ruled out.

As the worldwide number of nurses and physician developing COVID-19 increases, and the number of deaths among nurses and physicians continue to growth, it is very clear that our traditional approach has drastically failed to protect HCWs. Among the several reasons for this failure is the fact that patients with COVID-19 may arrive to the hospital with atypical presentations (e.g. diarrhea), and as we keep learning about COVID-19 we continue to find new ways that this disease may initially present (e.g. anosmia). Our traditional approach to isolate only suspected patients is failing primarily because we are failing to suspect the disease in patients with non-classical clinical presentations.

At the University of Louisville, we started a COVID19 surveillance program in all ten hospitals in the city.[1] We are currently testing patients admitted to the Louisville hospitals with signs and symptoms of respiratory infection or gastrointestinal infection. We have tested 2,900 patients with 342 positive tests. We now suggest that universal test-and-isolate for all hospitalized patients should be our new approach to protect HCWs. An algorithm to implement this new approach is depicted in Figure 1.

According to this approach, at the time of hospital admission all patients will have a nasopharyngeal swab performed for SARS-CoV-2 identification by reverse- transcriptase polymerase-chain-reaction (RT-PCR). If the patient has no risk factors for COVID-19, the patient will be placed in contact and droplet isolation. If the patient has risk factors for COVID-19, the patient will be placed in contact and airborne isolation. According to the number and types of risk factors, patients should be classified as being low risk versus high risk for COVID19. We suggest that a local COVID-19 multidisciplinary clinical team perform stratification of patients as being a) No Risk, b) Low Risk, and c) High Risk. There are no established criteria to define a patient as high risk, but a patient hospitalized with pneumonia with a characteristic computed tomography (CT) showing bilateral ground-glass opacities should always be considered a high risk patient.

Patients with no risk factors who test negative for COVID-19 can be removed from isolation. On the other hand, in patients with risk factors for COVID-19, a nasopharyngeal swab with a negative PCR for SARSCoV-2 should not be used as the single criteria to discontinue isolation. The determination to discontinue isolation should be based on whether the patient has been stratified into the high-risk group versus the low risk group. If the patient is low risk and tests negative, isolation may be discontinued. If the patient is at high risk for COVID-19 and a nasopharyngeal swab is negative for SARS-CoV-2 the patient should remain in contact and airborne isolation. We have witnessed two patients with classical CT findings with negative nasopharyngeal swab RT-PCR, but positive RT-PCR for SARS-CoV-2 in sputum samples.

In addition to universal test-and-isolation all patients at time of hospitalization, we suggest that all patients who develop cough, shortness of breath, hypoxemia, or pneumonia during hospitalization be retested. Since COVID-19 may have an incubation period of several 


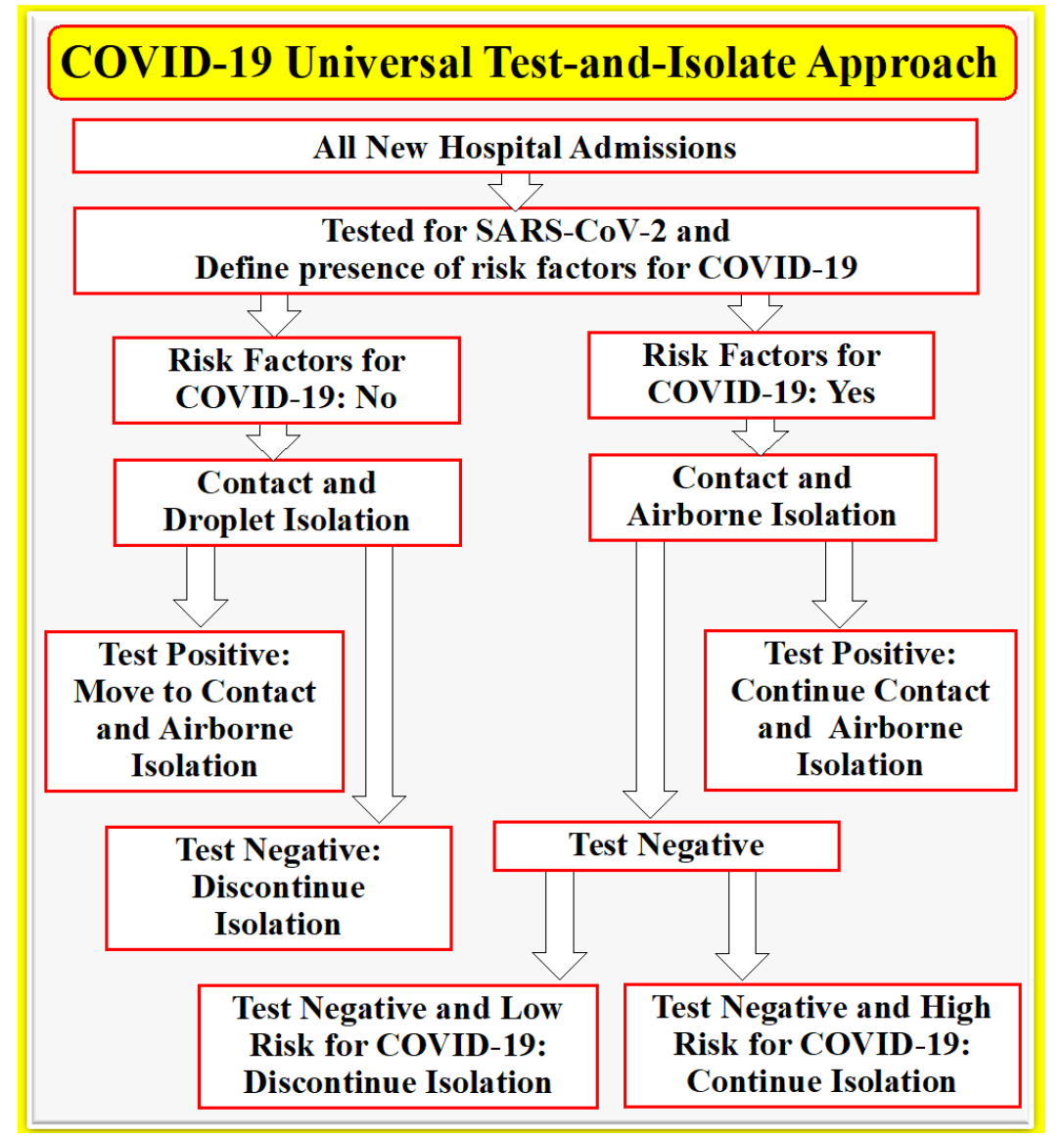

Figure 1. Suggested algorithm to implement universal test-and-isolate for all hospitalized patients.

days or weeks, any patient who develops pneumonia during hospitalization should be tested for COVID-19. We have witnessed a patient who developed pneumonia seven days after hospital admission, with classical CT scan finding for COVID-19 and a positive nasopharyngeal swab RT-PCR for SARS-CoV-2.
In conclusion, we believe that the implementation of a universal test-and-isolate for all newly admitted patients might be a critical measure to help prevent COVID-19 in nurses, physicians, and other HCWs.
Published: April 8, 2020

Copyright: (c) 2022 The author(s). This original article is brought to you for free and open access by ThinkIR: The University of Louisville's Institutional Repository. For more information, please contact thinkir@louisville.edu. This article is distributed under the terms of the Creative Commons Attribution 4.0 International License (CC BY 4.0), which permits un- restricted use, distribution, and reproduction in any medium, provided the original author and source are credited.

Funding Source: The author(s) received no specific funding for this work.

Conflict of Interest: All authors declared no conflict of interest in relation to the main objective of this work.

\section{References}

1. Ramirez JA, Palmer KE, Carrico R, Arnold FW, Chung D, Wolf LA. Louisville Coronavirus Surveillance Program. Univ Louisville J Respir Infect 2020; 4(1):Article 3. doi: 10.18297/jri/vol4/iss1/3. 